\section{Custos dos mutirões de mamografia de 2005 e 2006 na Direção Regional de Saúde de Marília, São Paulo, Brasil}

\author{
Costs of mammogram campaigns in the Regional \\ Health Division of Marília, São Paulo State, Brazil, \\ 2005-2006
}

${ }^{1}$ Escola Paulista de Medicina, Universidade Federal de São Paulo, São Paulo, Brasil.

2 Departamento Regional de Saúde de Marília, Marília, Brasil.

Correspondência R. R. F. Marconato Departamento Regional de Saúde de Marília. Rua Doutor Cesar Martins Pirajá 660, Marília, SP 17502-170, Brasil. roselifreire@hotmail.com

\section{Abstract}

The aim of this study was to measure the direct costs of mammogram campaigns conducted by the Regional Health Division of Marília, São Paulo State, Brazil, in 2005 and 2006. A total of 11,952 mammograms were performed. Mammographic outcomes were classified according to BI-RADS. Cost analysis was based on the amount paid by the Brazilian Unified National Health System (SUS). Ten cases of breast cancer were diagnosed (0.84 per 1,000 mammograms), 70\% of which between 50 and 69 years of age. The campaigns and follow-up cost a total of $R \$ 450,019$ (U\$280,000), with $R \$ 431,467$ paid for 11,952 mammograms and $R \$ 18,552$ for diagnosing 29 suspected cases and treating 3 cases of benign tumors and 6 cancer cases. Mean cost per diagnosed case was $R \$ 43,268$. The high cost per diagnosed case highlights the need to implement effective screening programs and improve the quality of mammography services in this region of São Paulo State.

Breast Neoplasms; Mammography; Costs and Cost Analysis
Roseli Regina Freire Marconato 1,2

Patrícia Coelho de Soárez 1

Rozana Mesquita Ciconelli 1

\section{Introdução}

O câncer de mama é considerado um importante problema de saúde pública em todo o mundo. Estima-se que mais de um milhão de mulheres são diagnosticadas com câncer de mama a cada ano, e mais de 410 mil morrerão da doença. Em países menos desenvolvidos a infraestrutura e recursos para mamografias de rotina muitas vezes não são disponíveis ocasionando o diagnóstico de cânceres de mama em estágios mais avançados 1 .

O número de casos novos de câncer de mama esperados para o Brasil, no ano de 2010, é de 49.240, com um risco estimado de 49 casos a cada 100 mil mulheres. Na Região Sudeste é o mais incidente entre as mulheres com um risco de 64 casos novos por 100 mil e no Estado de São Paulo é de 68 a cada 100 mil mulheres e corresponde a $21,72 \%$ do total de cânceres 2 .

$\mathrm{O}$ rastreamento do câncer de mama com mamografia e o investimento em mamografia de alta qualidade possibilita o diagnóstico do câncer de mama em sua fase pré-clínica reduzindo a mortalidade mulheres com esta doença ${ }^{3}$. Ensaios clínicos randomizados que comparam a mortalidade em mulheres que participaram de rastreamento mamográfico com mulheres não submetidas a nenhuma intervenção são favoráveis ao uso da mamografia como método de detecção precoce capaz de reduzir a mortalidade por câncer de mama. Estudos de meta-análise 
demonstram que os benefícios do uso da mamografia se referem, principalmente, a cerca de $30 \%$ de diminuição da mortalidade em mulheres acima dos 50 anos, depois de sete a nove anos de implementação de ações organizadas de rastreamento (Instituto Nacional de Câncer. Detecção precoce do câncer de mama. http://wwww.inca. gov.br/conteudo_view.asp?id=1932, acessado em Out/2009).

No Estado de São Paulo os dados do Registro Hospitalar de Câncer (Secretaria de Estado da Saúde de São Paulo. Banco de dados. http:/ / www. fosp.saude.sp.gov.br, acessado em Jul/ 2010), disponibilizados pela Fundação Oncocentro de São Paulo (FOSP), demonstram que a detecção precoce do câncer de mama é pequena, pois segundo o estadiamento do câncer de mama no biênio $2007 / 2008$, apenas $7,06 \%$ dos tumores referemse aos tumores in situ (estadiamento 0) número bastante inferior ao encontrado, por exemplo, no Canadá, que tem um programa organizado de rastreamento, cujo percentual é de $29,9 \% 4$.

Em virtude disso, a Secretaria Estadual de Saúde de São Paulo (SES-SP) tem promovido semestralmente, desde 2005, mutirões para realização de exames de mamografia no âmbito das unidades de saúde do Estado de São Paulo, destinados ao atendimento de todas as mulheres com solicitação de realização de mamografia que não tenham conseguido agendar ou realizar seu exame, tendo como objetivo principal eliminar a demanda reprimida para a execução do procedimento.

$\mathrm{Na}$ literatura internacional existem estudos demonstrando a redução do custo do tratamento do câncer de mama quando detectado em estádios iniciais. No Brasil o estudo de Kemp et al. 5, de estimativa de custos incluindo o rastreamento mamográfico, demonstra a redução dos custos quando incorporada a mamografia de rastreamento, mas há ainda uma lacuna de estudos sobre custos diretos envolvendo rastreamento mamográfico em massa, como é o caso dos mutirões de mamografia desenvolvidos no Estado de São Paulo.

Os custos de todo o ciclo do mutirão que incluem o rastreamento, os procedimentos adicionais até o diagnóstico e o tratamento do câncer de mama são fundamentais para avaliar a aplicabilidade dessa estratégia como política de saúde pública. Neste sentido este estudo tem como objetivo apresentar os custos diretos dos mutirões realizados na região da Direção Regional (DIR) de Saúde de Marília nos anos de 2005 e 2006.

\section{Materiais e métodos}

A DIR de saúde de Marília, região administrativa da Secretaria de Estado da Saúde de São Paulo, localiza-se a centro-oeste do Estado de São Paulo, compreende 37 municípios atualmente agrupados em 3 microrregiões de saúde: Adamantina, Marília e Tupã. Em 2006, sua população estimada segundo o Instituto Brasileiro de Geografia e Estatística (IBGE) era de 618.045 com 311.403 mulheres e em idade fértil, 195.571 (Departamento de Informática do SUS. Informações de saúde demográficas e socioeconômicas - projeções intercensitárias. http://www. datasus.gov.br/DATASUS/index.php, acessado em 19/Dez/2009). A rede de alta complexidade em oncologia nesta região é composta por três serviços, sendo duas Unidades de Alta Complexidade em Oncologia, unidades que oferecem o tratamento cirúrgico do câncer e quimioterapias, sendo uma em Marília e uma em Tupã e um Centro de Alta Complexidade em Oncologia, que além do tratamento cirúrgico, quimioterapia, também conta com radioterapia, em Marília.

No período de 2005-2006 aconteceram quatro mutirões. Os exames de mamografia foram realizados em oito serviços de radiologia, cinco deles estão localizados em Marília, dois em Tupã e um em Pompéia.

Os resultados das mamografias foram classificados de acordo com as categorias do sistema Breast Imaging Reporting and Data System (BI-RADS), enumerando os achados de 0 a 5 e os correlacionando a uma conduta clínica a ser seguida, assim descrita: BI-RADS 0: avaliação inconclusiva - necessita de estudo adicional; BIRADS 1: normal; BI-RADS 2: achados benignos; BI-RADS 3: achados provavelmente benignos (controle em 6-12-24 meses desde que sem alterações apreciáveis); BI-RADS 4: alterações suspeitas (considerar prosseguir investigação citohistológica) e BI-RADS 5: alterações altamente suspeitas (prosseguir investigação) 6 .

A orientação da Secretaria de Estado da Saúde nestes mutirões foi para que as pacientes que tiveram classificação da mamografia nas categorias BI-RADS 4 e 5 fossem agendadas em serviço de referência em oncologia para investigação diagnóstica e tratamento.

Os dados foram coletados dos documentos gerados pelo mutirão, que estão sob a guarda da área de compra de serviços do Departamento Regional de Saúde de Marília (DRS 9). O primeiro documento, de preenchimento obrigatório pelo serviço que realizou a mamografia, apresenta os dados de identificação da mulher, a data de realização do exame, data de realização da última mamografia e resultado da mamografia 
pela classificação BI-RADS. O segundo documento é o de acompanhamento das pacientes com classificação de BI-RADS 4 e 5 preenchido pela área técnica da DIR de Saúde, apresenta data do mutirão, nome do serviço que realizou a mamografia, idade da paciente, município de residência, classificação BI-RADS, local de atendimento (serviço de oncologia), procedimento realizado, diagnóstico clínico ou anatomo-patológico e tipo de tratamento adotado. O detalhamento dos procedimentos realizados no tratamento das pacientes foi fornecido pelos serviços de referência em oncologia que realizaram o tratamento, com até dois anos de acompanhamento, cujos custos das internações para o procedimento cirúrgico foram retirados dos espelhos das internações hospitalares do sistema de faturamento do DRS.

Os custos analisados foram os valores pagos pela Secretaria de Estado pelos exames de mamografia aos serviços contratados, e dos procedimentos realizados nas pacientes diagnosticadas com BI-RADS 4 e 5, que tiveram acompanhamento após os mutirões nos serviços de referência, pagos pelo Sistema Único de Saúde (SUS).

Foram incluídos no estudo os seguintes custos diretos médicos: consultas médicas especializadas, internações, atendimentos de urgência, e procedimentos de apoio diagnóstico e terapêutico fornecidos pelo serviço que realizou o procedimento. Para determinação dos custos dos procedimentos foram utilizados os valores da Tabela de procedimentos do Sistema de Informação Ambulatorial SIA/SUS versão Abril de 2005 e da Tabela de procedimentos do Sistema de Informação Hospitalar SIH/SUS Versão Abril de 2005.

A identificação dos recursos utilizados e posterior cálculo dos custos foram feitos através da construção dos seguintes indicadores: custo total do mutirão (CTM), custo médio de acompanhamento (CMA) e custo médio para detecção dos casos de câncer (CMDC), que foram calculados com as seguintes fórmulas:

CTM = Valor do Mutirão + Valor de Acompanhamento do BI-RADS 4 e 5 (VA B4 B5)

$\Sigma$ do custo de cada procedimento realizado (consultas, exames, internações, etc.) no. de BI-RADS 4 e 5 acompanhados

CMDC $=\frac{\text { CTM }}{\text { no. de casos de câncer detectados }}$

Este estudo teve a autorização da Diretoria do DRS 9 e a aprovação do Comitê de Ética em Pesquisa da Universidade Federal de São Paulo (UNIFESP)/Hospital São Paulo.

\section{Resultados}

Foram realizados 11.952 exames de mamografia em quatro mutirões, nos meses de maio e novembro de 2005 e maio e novembro de 2006, em 8 serviços de radiologia, contratados especificamente para o mutirão. As mulheres que realizaram o exame tinham solicitação médica de mamografia e eram residentes na área dos 37 municípios da região, foram 5.385 mamografias no mutirão de 2005 e 6.567 em 2006.

As mulheres de 40-49 anos foram as mais representativas nestes mutirões com 36,9\% de participação (4.410 mulheres), seguidas das de 50-59 anos com 30,76\% (3.677) e de 60-69 anos representaram $14,83 \%$ (1.773), conforme apresentado na Tabela 1.

A Tabela 2 apresenta o número de exames realizados e classificação por categoria BI-RADS. Os exames considerados alterados foram 32, sendo 28 da categoria BI-RADS 4 e 4 da categoria BIRADS 5, e estes foram alvo de acompanhamento nos serviços de oncologia de referência regional.

Foi confirmado diagnóstico de câncer de mama em 10 pacientes $(31,25 \%)$ das 32 com BIRADS 4 e 5, sendo 1 do mutirão de maio de 2005, 1 em novembro de 2005, 6 em maio de 2006 e 2 em novembro de 2006. Dos 10 casos confirmados $70 \%$ (7 mulheres) estavam na faixa etária de 50-69 anos. Dos 28 BI-RADS 4, considerados como suspeitos, 1 paciente não realizou o exame para diagnóstico e $8(28,57 \%)$ tiveram confirmação de câncer, dos casos de categoria BI-RADS 5, considerados como altamente suspeitos, 2 (50\%) tiveram confirmação de câncer de mama.

Para a Secretaria de Estado da Saúde, com o custo unitário da mamografia de $\mathrm{R} \$ 36,10$ (valor de tabela SIA/SUS), o valor do mutirão (VM) foi de $\mathrm{R} \$ 431.467,20$ referente ao pagamento das 11.952 mamografias realizadas nos mutirões de 2005 e 2006.

As 32 mulheres com resultado dos exames com BI-RADS 4 e 5 foram encaminhadas para acompanhamento em 4 serviços de referência em oncologia, destas, 29 foram encaminhadas para 3 serviços na região da DIR de Marília, e 3 foram atendidas em um serviço na região da DIR de Bauru. As informações dos procedimentos realizados nos atendimentos destas mulheres só puderam ser obtidas nos serviços de referência da DIR Marília. Portanto, das três mulheres atendidas no serviço da DIR de Bauru foi disponibilizada apenas a informação do diagnóstico, tendo uma delas a confirmação de câncer.

Nos dez casos de câncer diagnosticados, uma das mulheres realizou o tratamento no serviço de oncologia da DIR de Bauru, onde não foram 
disponibilizadas informações sobre os procedimentos realizados. Dois casos provavelmente realizaram a cirurgia em caráter particular ou por convênio médico, pois não há internação pelo SUS e uma paciente apesar de confirmado o diagnóstico até o levantamento dos dados tinha abandonado o tratamento, não realizando a cirurgia. Portanto, foi possível ter acesso ao procedimento cirúrgico realizado em 6 pacientes com câncer.

A Tabela 3 descreve os procedimentos que foram realizados no acompanhamento e tratamento das mulheres com BI-RADS 4 e 5, perfazendo um valor total de $\mathrm{R} \$ 18.552,71$. Neste acompanhamento foram realizados 9 procedimentos cirúrgicos, dos quais 3 foram em mulheres com lesões benignas e 6 com tumores malignos. Foram gastos com procedimentos para confirmação diagnóstica R\$1.213,84.

Há registro de quimioterapia de 5 pacientes com o pagamento SUS e de radioterapia de 2 pacientes. A realização de radioterapia só foi obtida das pacientes que fizeram todo o seguimento no serviço que é um centro de alta complexidade em oncologia e tem serviço de radioterapia.

O gasto com o acompanhamento e tratamento das pacientes com câncer (confirmação diagnóstica, cirurgia, quimioterapia e radioterapia) foi de $\mathrm{R} \$ 16.536,81$ variando de $\mathrm{R} \$ 405,57$ a $\mathrm{R} \$$ $4.399,44$, com um valor médio de $\mathrm{R} \$ 2.706,71$ ( Tabela 4). O maior gasto foi com quimioterapia no valor de $\mathrm{R} \$ 9.450,65$, representando $57,15 \%$ do valor total.

Foi gasto com pacientes que não tiveram confirmação de câncer de mama o valor de $\mathrm{R}$ \$ $2.015,90$, dos quais $\mathrm{R} \$ 669,16$ com procedimentos cirúrgicos.

O custo total do mutirão de mamografia nos anos de 2005 e 2006 na região de saúde da DIR Marília foi de R\$450.019,91 (R\$ 431.467,20 com o rastreamento e $\mathrm{R} \$ 18.552,71$ com o tratamento).

O custo médio de acompanhamento foi de $\mathrm{R} \$ 598,47(18.552,71 / 31)$ por caso de BI-RADS 4 ou 5 acompanhado.

O custo médio para cada caso de câncer detectado foi de $\mathrm{R} \$ 45.001,99$ ( $\mathrm{R} \$ 450.019,91 / 10)$.

\section{Discussão}

Neste estudo foi possível descrever os mutirões de mamografia desenvolvidos pela SES-SP na região da DIR de Marília constatando um aumento de exames de um ano para o outro (5.385 em 2005 e 6.567 em 2006) demonstrando um aumento na cobertura.

A utilização da classificação por categoria do BI-RADS permitiu a padronização dos resultados
Tabela 1

Número de mamografias realizadas por faixa etária segundo mutirões na região da Direção Regional de Saúde de Marília, São Paulo, Brasil, em 2005 e 2006.

\begin{tabular}{|c|c|c|}
\hline \multirow[t]{2}{*}{ Faixa etária/Mutirão } & \multicolumn{2}{|c|}{ Total } \\
\hline & $\mathrm{n}$ & $\%$ \\
\hline \multicolumn{3}{|l|}{ Até 39 anos } \\
\hline Maio/2005 & 307 & 23,12 \\
\hline Novembro/2005 & 294 & 22,14 \\
\hline Maio/2006 & 265 & 19,95 \\
\hline Novembro/06 & 462 & 34,79 \\
\hline Total & 1.328 & 11,11 \\
\hline \multicolumn{3}{|l|}{ 40-49 anos } \\
\hline Maio/2005 & 1.244 & 28,21 \\
\hline Novembro/2005 & 758 & 17,19 \\
\hline Maio/2006 & 987 & 22,38 \\
\hline Novembro/2006 & 1.421 & 32,22 \\
\hline Total & 4.410 & 36,90 \\
\hline \multicolumn{3}{|l|}{ 50-59 anos } \\
\hline Maio/2005 & 1.031 & 28,04 \\
\hline Novembro/2005 & 607 & 16,51 \\
\hline Maio/2006 & 925 & 25,16 \\
\hline Novembro/2006 & 1.114 & 30,30 \\
\hline Total & 3.677 & 30,76 \\
\hline \multicolumn{3}{|l|}{$60-69$ anos } \\
\hline Maio/2005 & 461 & 26,00 \\
\hline Novembro/2005 & 312 & 17,60 \\
\hline Maio/2006 & 435 & 24,53 \\
\hline Novembro/2006 & 565 & 31,87 \\
\hline Total & 1.773 & 14,83 \\
\hline \multicolumn{3}{|l|}{70 anos ou mais } \\
\hline Maio/2005 & 170 & 27,78 \\
\hline Novembro/2005 & 99 & 16,18 \\
\hline Maio/2006 & 152 & 24,84 \\
\hline Novembro/2006 & 191 & 31,21 \\
\hline Total & 612 & 5,12 \\
\hline \multicolumn{3}{|l|}{ Não informado } \\
\hline Maio/2005 & 69 & 45,39 \\
\hline Novembro/2005 & 33 & 21,71 \\
\hline Maio/2006 & 27 & 17,76 \\
\hline Novembro/2006 & 23 & 15,13 \\
\hline Total & 152 & 1,27 \\
\hline Total & 11.952 & 100,00 \\
\hline
\end{tabular}

e a adoção de acompanhamento estabelecidas no mutirão, bem como possibilitou a comparação com outros estudos.

A quantidade identificada nesses mutirões de casos suspeitos e altamente suspeitos (categoria BI-RADS 4 e 5) foi abaixo do esperado descrito 
Tabela 2

Classificação dos resultados das mamografias realizadas segundo mutirões na região da Direção Regional de Saúde de Marília, São Paulo, Brasil, 2005 e 2006.

\begin{tabular}{|c|c|c|c|c|c|c|c|c|c|c|}
\hline \multirow[t]{3}{*}{ Resultado } & \multicolumn{10}{|c|}{ Mutirões } \\
\hline & \multicolumn{2}{|c|}{ Maio/2005 } & \multicolumn{2}{|c|}{ Novembro/2005 } & \multicolumn{2}{|c|}{ Maio/2006 } & \multicolumn{2}{|c|}{ Novembro/2006 } & \multicolumn{2}{|c|}{ Total } \\
\hline & $\mathbf{n}$ & $\%$ & $\mathrm{n}$ & $\%$ & $\mathbf{n}$ & $\%$ & $\mathbf{n}$ & $\%$ & $\mathbf{n}$ & $\%$ \\
\hline BI-RADS 0 & 275 & 8,38 & 187 & 8,89 & 252 & 9,03 & 403 & 10,67 & 1.117 & 9,35 \\
\hline BI-RADS 1 & 1.427 & 43,48 & 927 & 44,08 & 1132 & 40,56 & 1.727 & 45,74 & 5.213 & 43,62 \\
\hline BI-RADS 2 & 1.493 & 45,49 & 935 & 44,46 & 1304 & 46,72 & 1.556 & 41,21 & 5.288 & 44,24 \\
\hline BI-RADS 3 & 81 & 2,47 & 49 & 2,33 & 90 & 3,22 & 82 & 2,17 & 302 & 2,53 \\
\hline BI-RADS 4 & 6 & 0,18 & 3 & 0,14 & 12 & 0,43 & 7 & 0,19 & 28 & 0,23 \\
\hline BI-RADS 5 & 0 & 0,00 & 2 & 0,10 & 1 & 0,04 & 1 & 0,03 & 4 & 0,03 \\
\hline Total & 3.282 & 27,46 & 2103 & 17,60 & 2.791 & 23,35 & 3.776 & 31,59 & 11.952 & 100,00 \\
\hline
\end{tabular}

BI-RADS: Breast Imaging Reporting and Data System; BI-RADS 0: avaliação inconclusiva - necessita de estudo adicional; BI-RADS 1: normal; BI-RADS 2: achados benignos; BI-RADS 3: achados provavelmente benignos (controle em 6-12-24 meses desde que sem alterações apreciáveis); BI-RADS 4: alterações suspeitas (considerar prosseguir investigação cito-histológica) e BI-RADS 5: alterações altamente suspeitas (prosseguir investigação).

\section{Tabela 3}

Procedimentos realizados nas pacientes com BI-RADS 4 e 5 dos mutirões de 2005 e 2006 na região da Direção Regional de Saúde de Marília, São Paulo, Brasil.

\begin{tabular}{|c|c|c|c|}
\hline Procedimentos realizados & Quantidade & Valor unitário & Valor (R\$) \\
\hline Administração de medicamentos & 4 & 0,63 & 2,52 \\
\hline Atendimento médico com obs. até 24 horas & 1 & 12,47 & 12,47 \\
\hline Atendimento médico em urgência/emergência & 6 & 8,16 & 48,96 \\
\hline Consulta/atendimento em assistência especializada e alta & 7 & 2,55 & 17,85 \\
\hline Consultas médicas especializadas & 132 & 7,55 & 996,60 \\
\hline Exame anatomo-patológico para biopsia ou biopsia aspirativa & 7 & 20,87 & 146,09 \\
\hline Exames de patologia clínica & 153 & 2,71 * & 414,73 \\
\hline Exames de raios $X$ simples & 13 & 9,53 * & 123,95 \\
\hline Ecografia de abdômen total & 2 & 21,53 & 43,06 \\
\hline Ecografia de mama & 13 & 13,34 & 173,42 \\
\hline Eletrocardiograma & 6 & 3,2 & 19,20 \\
\hline Mamografias & 17 & 36,1 & 613,70 \\
\hline Prova de função pulmonar & 1 & 3,18 & 3,18 \\
\hline Punções de mama & 19 & 14,77 & 280,63 \\
\hline Procedimentos cirúrgicos & 9 & 399,44 * & $3.567,98$ \\
\hline Quimioterapia & 53 & 178,31 * & $9.450,65$ \\
\hline Radioterapia & 153 & 17,24 * & $2.637,72$ \\
\hline Total & 596 & & $18.552,71$ \\
\hline
\end{tabular}

* Valor médio. 
Valores pagos pelo SUS em pacientes com câncer dos mutirões de 2005 e 2006 por serviço de referência na região da Direção Regional de Saúde de Marília, São Paulo, Brasil.

\begin{tabular}{|c|c|c|c|c|c|c|c|c|c|c|}
\hline \multirow[t]{3}{*}{ Procedimentos } & \multicolumn{9}{|c|}{ Serviços de referência } & \multirow[t]{3}{*}{ Total } \\
\hline & \multicolumn{4}{|c|}{$\begin{array}{l}\text { Centro de alta complexidade } \\
\text { em oncologia }\end{array}$} & \multicolumn{3}{|c|}{$\begin{array}{l}\text { Unidades de alta } \\
\text { complexidade em oncologia } \\
\text { (I) }\end{array}$} & \multicolumn{2}{|c|}{$\begin{array}{l}\text { Unidade em alta } \\
\text { complexidade em } \\
\text { oncologia (II) }\end{array}$} & \\
\hline & 1 & 2 & 3 & 4 & 5 & 6 & 7 & 8 & 9 & \\
\hline Administração de medicamentos & 1,26 & 0,00 & 0,00 & 0,00 & 0,00 & 0,00 & 0,00 & 0,00 & 0,00 & 1,26 \\
\hline $\begin{array}{l}\text { Atendimento médico em urgência/ } \\
\text { emergência }\end{array}$ & 24,48 & 8,16 & 0,00 & 0,00 & 0,00 & 0,00 & 0,00 & 0,00 & 0,00 & 32,64 \\
\hline $\begin{array}{l}\text { Consulta/atendimento em assistência } \\
\text { especializada e alta }\end{array}$ & 12,75 & 5,10 & 0,00 & 0,00 & 0,00 & 0,00 & 0,00 & 0,00 & 0,00 & 17,85 \\
\hline Consultas médicas especializadas & 158,55 & 75,50 & 60,40 & 15,10 & 30,20 & 75,50 & 45,30 & 45,30 & 37,75 & 543,60 \\
\hline Ecografia de abdômen total & 43,06 & 0,00 & 0,00 & 0,00 & 0,00 & 0,00 & 0,00 & 0,00 & 0,00 & 43,06 \\
\hline Ecografia de mama & 13,34 & 26,68 & 26,68 & 13,34 & 13,34 & 0,00 & 0,00 & 0,00 & 0,00 & 80,04 \\
\hline Eletrocardiograma & 3,20 & 3,20 & 3,20 & 0,00 & 0,00 & 0,00 & 0,00 & 0,00 & 0,00 & 9,60 \\
\hline $\begin{array}{l}\text { Exame anatomo-patológico para } \\
\text { biopsia ou biopsia aspirativa }\end{array}$ & 0,00 & 0,00 & 0,00 & 0,00 & 0,00 & 0,00 & 0,00 & 20,87 & 104,35 & 125,22 \\
\hline Exames de patologia clínica & 89,48 & 74,35 & 54,04 & 0,00 & 15,12 & 7,81 & 0,00 & 31,38 & 26,84 & 299,02 \\
\hline Exames de raios $X$ simples & 45,33 & 19,00 & 19,00 & 0,00 & 9,50 & 0,00 & 0,00 & 9,50 & 9,50 & 111,83 \\
\hline Mamografias & 72,20 & 0,00 & 0,00 & 0,00 & 0,00 & 36,10 & 0,00 & 0,00 & 0,00 & 108,30 \\
\hline Punções de mama & 0,00 & 0,00 & 0,00 & 0,00 & 73,85 & 0,00 & 103,39 & 0,00 & 0,00 & 177,24 \\
\hline Procedimentos cirúrgicos & 508,67 & 892,45 & 298,52 & 0,00 & 559,52 & 0,00 & 0,00 & 298,52 & 341,14 & $2.898,82$ \\
\hline Quimioterapia & $1.519,65$ & 853,60 & $2.774,20$ & 0,00 & $3.236,20$ & 0,00 & 0,00 & 0,00 & $1.067,00$ & $9.450,65$ \\
\hline Radioterapia & 0,00 & $1.474,28$ & $1.163,40$ & 0,00 & 0,00 & 0,00 & 0,00 & 0,00 & 0,00 & $2.637,68$ \\
\hline Total & $2.478,63$ & $3.432,32$ & $4.399,44$ & 28,44 & $3.937,73$ & 119,41 & 148,69 & 405,57 & $1.586,58$ & $16.536,81$ \\
\hline
\end{tabular}

na literatura e também inferior ao mutirão do Estado como um todo, o que influenciou no baixo número de câncer de mama encontrado.

Neste estudo a proporção de câncer para cada 1.000 exames de mamografia realizados foi de 0,84 casos. O documento Mamografia: Da Prática ao Controle 8 e o estudo de Benveniste et al. 7 indicam que o índice de detecção de câncer de mama pelo rastreamento mamográfico é estimado em 6-10 casos por 1.000 exames, se considerar apenas tumores prevalentes, encontrados na primeira mamografia de rotina, e de 2-4 casos por 1.000 nos exames subsequentes (cânceres incidentes). O relatório dos mutirões de mamografias no Estado de São Paulo indica que são esperados aproximadamente de 5-8 casos de câncer de mama em 1.000 mulheres em programas de rastreamento 6 .

O valor despendido pela SES-SP para pagamento de 11.952 mamografias foi de $\mathrm{R} \$$ $431.467,20$. Para a investigação diagnóstica de 29 casos suspeitos (categoria BI-RADS 4 e 5) e tratamento de 3 casos de tumores benignos e 6 casos de câncer, o Ministério da Saúde gastou $\mathrm{R} \$ 18.552,71$, totalizando R\$ 450.019,91.

Esses mutirões não previram o acompanhamento das pacientes com categoria BI-RADS 3 (achado provavelmente benigno) e da categoria 0 (avaliação adicional) o que pode ter deixado de diagnosticar muitos casos de câncer, considerando que do BI-RADS 3 o esperado é de $3 \%$ com câncer e de BI-RADS 0 é de $13 \%$.

$\mathrm{O}$ custo para o diagnóstico nesses mutirões foi de $\mathrm{R} \$ 432.681,04(\mathrm{R} \$ 431.467,20+\mathrm{R} \$ 1.213,84)$ o que representa $\mathrm{R} \$ 43.268,10$ por diagnóstico se considerarmos 10 casos de câncer confirmados. Este valor está muito acima do descrito no estudo de Kemp et al. 5 que obteve o custo de $\mathrm{R} \$ 15.318,75$ por diagnóstico, utilizando a tabela Associação Médica Brasileira (AMB) 92, em que os valores pagos são muito superiores aos pagos pela Tabela SUS. O custo da mamografia na tabela AMB 92 é de $R \$ 62,00$, enquanto na tabela SIA-SUS é de R\$ 36,10, a remuneração SUS corresponde a $58 \%$ da remuneração da AMB, no entanto, observa-se que esta relação não é igual 
para todos os procedimentos, havendo diferenças significativas entre os procedimentos. Esta grande diferença de custo por caso detectado se dá pelo número reduzido de câncer de mama diagnosticado nesses mutirões. Comparando-se com o programa de rastreamento da Itália em que cada câncer diagnosticado apresenta um custo de 5.548 euros 4 o custo por diagnóstico de câncer nestes mutirões foi 3,5 vezes maior.

O custo dos tratamentos das 6 pacientes com câncer, incluindo confirmação diagnóstica, cirurgia, quimioterapia e radioterapia, foi de $\mathrm{R} \$$ 16.536, 81, variando de $\mathrm{R} \$ 405,57$ a $\mathrm{R} \$ 4.399,44$, com um valor médio de $\mathrm{R} \$ 2.706,71$. Os procedimentos cirúrgicos custaram $\mathrm{R} \$ 2.898,82$, a radioterapia de 2 pacientes custou $\mathrm{R} \$ 2.637,68$ (valor médio por paciente $\mathrm{R} \$ 1.318,84$ ) e a quimioterapia de 5 pacientes custou R\$ 9.450,65 (valor médio por paciente de $\mathrm{R} \$ 1.890,13)$.

Dos 6 tratamentos realizados apenas uma paciente não realizou quimioterapia, onde provavelmente o câncer diagnosticado encontrava-se em fase inicial da doença. A literatura aponta que a detecção do câncer de mama em fases iniciais propicia, mais frequentemente, o tratamento com cirurgias conservadoras, associadas ou não à radioterapia e a agentes anti-estrogênicos (tamoxifeno), reduzindo substancialmente o custo do tratamento 8 .

Kemp et al. 5, analisando o custo do atendimento e tratamento do câncer no estado de São Paulo no ano de 2002, afirmaram que se o diagnóstico ocorrer em fases mais iniciais (carcinoma in situ, estádios Ia e Ib), os gastos podem ser reduzidos em torno de $70 \%$ do total, já que o tratamento quimioterápico seria evitado.

Não foi possível neste estudo ter acesso aos exames histopatológicos das pacientes com confirmação diagnóstica do câncer de mama. No entanto, os tratamentos realizados envolvendo quimioterapia indicam que a maioria dos casos foram diagnosticados em estágios mais avançados da doença e, consequentemente com o custo do tratamento mais elevado.

Quando considerados os tratamentos realizados, o tratamento da paciente que não realizou quimioterapia foi de $\mathrm{R} \$ 405,57$ e se comparado aos que também não têm registro de radioterapia que apresentaram os valores de $\mathrm{R} \$ 2.478,63, \mathrm{R} \$$ 1.586,58 e R\$ 3.937,73 verifica-se uma diferença considerável. E verifica-se que o tipo de cirurgia realizado neste caso foi conservadora (setorectomia). O custo do tratamento que envolveu apenas uma cirurgia conservadora é muito inferior ao descrito por Kemp et al. 5, cujo valor médio da cirurgia é de $\mathrm{R} \$ 3.000,00$, diferença esta também atribuída ao valor inferior pago pela Tabela SUS.

Apesar de não termos a informação do custo do tratamento radioterápico de todas as pacientes, se estimarmos este custo pelo valor médio dos 2 tratamentos realizados, no valor de $\mathrm{R} \$$ $1.318,84$, teríamos que o custo do tratamento do câncer sem quimioterapia corresponde a $43 \%$ do valor dos tratamentos com maior complexidade. O que sugere que o gasto nestes mutirões foi mais elevado em função de os casos diagnosticados estarem em estádios mais avançados.

Além da questão econômica envolvida, existem questões importantes relacionadas principalmente à sobrevida e à qualidade de vida das mulheres submetidas ao rastreamento. Na literatura há estudos que relacionam pior qualidade de vida em mulheres submetidas à mastectomia quando comparadas àquelas submetidas à cirurgia conservadora da mama 9,10,11 e sabe-se que a detecção precoce do câncer de mama favorece as cirurgias conservadoras 12 . E ainda, o rastreamento do câncer tem como objetivo a detecção precoce propiciando um tratamento efetivo e consequentemente um aumento da sobrevida 13 .

Não houve exigências pela SES-SP em relação à qualidade dos serviços de mamografia, não verificando se possuem programa de controle de qualidade implantado, como uma forma de garantir que a imagem tenha valor diagnóstico, o que pode ter influenciado nos resultados obtidos. O alto custo de cada caso diagnosticado ( $\mathrm{R} \$$ $43.268,10$ ) aponta para a necessidade de implementação de programas de rastreamento efetivos e do controle da qualidade das mamografias nesta região do estado. 


\section{Resumo}

O objetivo desse estudo foi apresentar os custos diretos dos mutirões de mamografias realizados nos anos de 2005 e 2006 na região da Direção Regional de Saúde de Marília, São Paulo, Brasil. Foram realizadas 11.952 mamografias. A classificação do resultado da mamografia foi feita pelo sistema padronizado BI-RADS e a análise de custos foi baseada nos valores pagos pelo SUS com base nas Tabelas SIA/SUS e SIH/SUS de 2005. Foram diagnosticados 10 casos de câncer de mama (0,84 por 1.000 mamografias), 70\% estavam na faixa etária de 50-69 anos. O custo total desses mutirões e acompanhamento foi de $R \$ 450.019,91$, sendo $R \$$ 431.467,20 com o pagamento de 11.952 mamografias e $R \$ 18.552,71$ para a investigação diagnóstica de 29 casos suspeitos, para o tratamento de três casos de tumores benignos e de 6 casos de câncer. O custo de cada caso diagnosticado foi de $R \$ 43.268,10$. O alto custo de cada caso diagnosticado aponta para a necessidade de implementação de programas de rastreamento efetivos e de qualidade da mamografia nesta região do estado.

Neoplasias da Mama; Mamografia; Custos e Análise de Custo

\section{Colaboradores}

R. R. F. Marconato contribuiu na concepção e projeto, análise e interpretação dos dados, redação do Artigo e Aprovação final da versão a ser publicada. P. C. Soárez contribuiu na concepção e projeto, e aprovação final da versão a ser publicada. R. M. Ciconelli contribuiu na aprovação final da versão a ser publicada.

\section{Referências}

1. Coughlin SS, Ekwueme DU. Breast cancer as a global health concern. Cancer Epidemiol 2009; 33:315-8.

2. Instituto Nacional de Câncer. Estimativa 2010. Incidência de câncer no Brasil. Rio de Janeiro: Instituto Nacional de Câncer; 2009.

3. Duffy SW, Smith RA, Gabe R, Tabar L, Yen AM, Chen TH. Screening for breast cancer. Surg Oncol Clin N Am 2005; 14:671-97.

4. Secretaria de Estado da Saúde de São Paulo. O GAIS no Encontro Internacional sobre rastreamento de câncer de mama. Boletim Eletrônico do Grupo Técnico de Avaliação e Informações de Saúde 2009; (2):1-5.

5. Kemp C, Elias S, Gebrim LH, Nazário ACP, Baracat EC, Lima GR. Estimativa de custo do rastreamento mamográfico em mulheres no climatério. Rev Bras Ginecol Obstet 2005; 27:415-20.

6. Fundação Oncocentro de São Paulo. Relatório mutirões de mamografias no Estado de São Paulo. São Paulo: Fundação Onocentro de São Paulo; 2009.

7. Benveniste APA, Ferreira AHPG, Aguillar VLN. Dupla leitura no rastreamento mamográfico. Radiol Bras 2006; 39:85-9.

8. Instituto Nacional de Câncer. Mamografia: da prática ao controle. Recomendações para profissionais de saúde. Rio de Janeiro: Instituto Nacional de Câncer; 2007.
9. Kattlove H, Liberati A, Keeler E, Brook RH. Benefits and costs of screening and treatment for early breast cancer. Development of a basic benefit package. JAMA 1995; 273:142-8.

10. Irwig L, Bennetts A. Quality of life after breast conservation or mastectomy: a systematic review. Aust N Z J Surg 1997; 67:750-4.

11. Nissen MJ, Swenson KK, Ritz LJ, Farrell JB, Sladek ML, Lally RM. Quality of life after breast carcinoma surgery: a comparison of three surgical procedures. Cancer 2001; 91:1238-46.

12. Makluf ASD, Dias RC, Barra ADA. Avaliação da qualidade de vida em mulheres com câncer da mama. Rev Bras Cancerol 2006; 52:49-58.

13. Chagpar AB, McMasters KM. Trends in mammography and clinical breast examination: a population-based study. J Surg Res 2007; 140:214-9.

14. Smith RA, Saslow D, Sawyer KA, Burke W, Costanza ME, Evans 3rd WP, et al. American Cancer Society guidelines for breast cancer screening: update 2003. CA Cancer J Clin 2003; 53:141-69.

Recebido em 17/Dez/2010 Aprovado em 01/Jun/2011 